\title{
Effects of Fish Meat-derived Peptide and Dipeptides on Dexamethasone-induced Fatigue in Mice
}

\author{
Yusuke Iwasaki ${ }^{1, \#}$, Hiroyasu Sakai ${ }^{2, \#,}$, Maho Asami ${ }^{2}$, Satoko Kitora ${ }^{2}$, Hiroaki Naito ${ }^{2}$, \\ Risako Kon ${ }^{2}$, Nobutomo Ikarashi², Junzo Kamei ${ }^{1,2, *}$ \\ ${ }^{1}$ Department of Biopharmaceutics and Analytical Science, Hoshi University, 2-4-41 Ebara, Shinagawa-ku, Tokyo 142-5501, Japan \\ ${ }^{2}$ Department of Biomolecular Pharmacology, Hoshi University, 2-4-41 Ebara, Shinagawa-ku, Tokyo 142-5501, Japan \\ ${ }^{\#}$ These authors contributed equally to this work. \\ *Corresponding author: kamei@ hoshi.ac.jp
}

Received October 02, 2019; Revised November 28, 2019; Accepted December 12, 2019

\begin{abstract}
In patients with inflammatory diseases, exogenous glucocorticoids have become the most common cause of drug-induced muscle wasting. In this study, we showed that isoleucine-arginine (IR) and arginineisoleucine (RI) are the main dipeptides with antioxidant activity in fish meat-derived peptide extract (FMDP). To investigate the anti-fatigue effect of FMDP and the two dipeptides (IR or RI), dexamethasone (DEX)-treated mice performed a weighted forced swimming test. Despite no change in body weight, the shortened swim time after DEX administration returned to baseline levels following the administration of FMDP, IR, and RI. However, the swim time of naive mice cannot be extended with the administration of FMDP, IR, or RI. Our data suggest that FMDP, IR, and RI may have beneficial effects on DEX-induced fatigue in mice. Nevertheless, further research is required to determine the mechanism through which FMDP reduces fatigue.
\end{abstract}

Keywords: antioxidant activity, fish meat-derived peptide, dexamethasone, fatigue, electron spin resonance

Cite This Article: Yusuke Iwasaki, Hiroyasu Sakai, Maho Asami, Satoko Kitora, Hiroaki Naito, Risako Kon, Nobutomo Ikarashi, and Junzo Kamei, "Effects of Fish Meat-derived Peptide and Dipeptides on Dexamethasone-induced Fatigue in Mice." Journal of Food and Nutrition Research, vol. 7, no. 12 (2019): 821-826. doi: $10.12691 /$ jfnr-7-12-2.

\section{Introduction}

According to the data collated by the World Health Organization (WHO) in 2018, Japan has one of the longest lifespans in the world [1]. The aging Japanese population has become a serious problem due to the growing prevalence of lifestyle-related diseases such as cancer, diabetes, and hypertension. According to a report by the Ministry of Health, Labour, more than 10 million people suffered from hypertension in Japan prior to the year 2014.

Reactive oxygen species (ROS) play a crucial role in human physiological and pathophysiological processes [2]. ROS include superoxide anions $\left(\mathrm{O}_{2}^{-}\right)$, hydroxyl radicals $\left(\mathrm{OH}^{*}\right)$, and hydrogen peroxide $\left(\mathrm{H}_{2} \mathrm{O}_{2}\right)$. Excess ROS generation plays a role in the development of numerous pathological conditions such as cancer [3], hypertension [4], inflammatory bowel disease [5], and fatigue [6].

Corticosteroids (glucocorticoids) are hormones that are secreted from the adrenal cortex and are responsible for homeostasis. Since corticosteroids have strong antiinflammatory and immunosuppressive effects, they are widely used for the treatment of various inflammatory and autoimmune diseases. More than 60 years they were first incorporated into clinical applications, the details of the molecular mechanisms behind the action of corticosteroids are still unknown. Furthermore, side effects often become a problem during treatment with corticosteroids, which is why there are continued efforts to separate the therapeutic actions from the side. Although glucocorticoids are highly effective, some of the adverse effects they may produce include hyperglycemia, decreased immune function, and skeletal muscle weakness [7]. Administering high doses of glucocorticoids also causes muscular atrophy in humans and animals [8,9]. With the increasing use of glucocorticoids to treat inflammatory diseases, exogenous glucocorticoids have become the most common cause of drug-induced muscle wasting, which is a clinical problem [10]. Recently, Huang et al indicated that dexamethasone (DEX) elevates oxidative stress markers, protein carbonyl levels and malondialdehyde content (lipid peroxidation) in skeletal muscle which resulted in mitochondrial dysfunction [11].

Marine fish are often consumed as a source of food, and many potential nutraceutical and medicinal products have been found in a large number of marine fish species. Consequently, muscle proteins, peptides, collagen, gelatin, oil, and bones from fish have yielded bioactive compounds. Certain peptides, such as imidazole, have an anti-fatigue effect. Imidazole is the generic name for a dipeptide containing carnosine and anserine. It exists within the skeletal muscles of organisms that move for a long time such as migratory birds, bonito, tuna, and other 
migratory fish [12]. Therefore, imidazole dipeptide has promise as a treatment for fatigue. To identify new bioactive compounds against steroid-induced fatigue, we examined the effects of fish meat-derived peptide extract (FMDP) in this study.

\section{Materials and Methods}

\subsection{Fractionation of the Fish Meat Sample}

The FMDP $(1.0 \mathrm{~g})$ was dissolved in water $(100 \mathrm{~mL})$ and centrifuged at $5,000 \times \mathrm{g}$ for $5 \mathrm{~min}$. The supernatant was purified with hexanes $(100 \mathrm{~mL} \times 2)$ and ethyl acetate $(100 \mathrm{~mL} \times 2)$ to remove lipids. The water phase was evaporated prior to fractionation with high-performance liquid chromatography (HPLC) fraction. FMDP was provided as an in-kind gift from Suzuhiro Co. Ltd.

HPLC was performed using a Shimadzu 20A series HPLC system, which consisted of a pump, autoinjector, column oven, UV detector, and a fraction collector. The water phase fraction was separated using an Inertsil ODS 3 column $(150 \times 4.6 \mathrm{~mm}, 5 \mu \mathrm{m})$ at a flow rate of $0.5 \mathrm{~mL} / \mathrm{min}$ and a constant temperature of $40^{\circ} \mathrm{C}$. The chromatography was performed with a solvent gradient using $0.1 \%$ aqueous formic acid (solvent $\mathrm{A}$ ) and methanol containing $0.1 \%$ formic acid (solvent B). The gradient started with $5 \%$ solvent B, was increased to $20 \%$ within $10 \mathrm{~min}$, increased to $90 \%$ for $1 \mathrm{~min}$, was kept $3 \mathrm{~min}$ to wash a column, decreased $5 \%$ within $1 \mathrm{~min}$, and equilibrated for $5 \mathrm{~min}$.

\subsection{Electron Spin Resonance Measurement of Hydroxyl Radical}

The analysis of hydroxyl radicals $(\bullet \mathrm{OH})$ was carried out using an electron spin resonance (ESR) spectrometer (JES-RE1X, JEOL Co., Tokyo, Japan). The ESR spectrum was measured using the following parameters: a microwave frequency of $9.43 \mathrm{GHz}$, a magnetic field of $335.5 \pm 5 \mathrm{mT}$, a power level of $9 \mathrm{~mW}$, a modulation of $100 \mathrm{kHz}$, a time constant of $0.03 \mathrm{~s}$, and a sweep time of 30 $\mathrm{s}$. The spectra of the samples were scanned to record the signal intensities (peak-to-peak heights).

A typical incubation mixture consisted of phosphate buffer (50 mM, pH 7.4), POBN (10 mM), DMSO (10\%), analytes, and copper (II) sulfate pentahydrate $(1 \mathrm{mM})$ in a final volume of $0.3 \mathrm{~mL}$. Samples were taken after a reaction time of $30 \mathrm{~min}$ at $37^{\circ} \mathrm{C}$.

\subsection{Animals}

Male ICR mice (6 weeks old, 20-23 g) were used for all experiments. All procedures were approved by the Animal Care Committee at Hoshi University (Tokyo, Japan).

\subsection{Treatment Protocol}

The following treatments were administered orally at Days 0,1, and 2: 100 and $300 \mathrm{mg} / \mathrm{kg}$ FMDP, 1 and $3 \mathrm{mg} / \mathrm{kg}$ dipeptide (arginine-isoleucine; RI or isoleucine-arginine;
IR) or the vehicle control ( $0.5 \%$ carboxymethyl cellulose; $\mathrm{CMC}$ ). Naïve mice did not receive any treatment. Mice were given a single subcutaneous (s.c.) injection of water-soluble DEX (15 mg/kg, Sigma Aldrich) once a day for three days, and saline was used as the vehicle control. On Days 0, 1, and 2, the $300 \mathrm{mg} / \mathrm{kg}$ FMDP, $3 \mathrm{mg} / \mathrm{kg}$ dipeptide (arginine-isoleucine; RI or isoleucine-arginine; IR) or the vehicle control (saline) were administered orally 15 min prior to DEX treatment.

\subsection{Weighted Forced Swimming Test}

Sixty minutes after treatment with FMDP, RI, and IR on Day 0 and Day 2, the mice were placed individually in a swimming pool filled with water $\left(24 \pm 1^{\circ} \mathrm{C}\right)$ to a depth of $15 \mathrm{~cm}$ with a lead sheath $(3 \%$ of the mouse's body weight) attached to the root of the tail. The swim time was recorded as the moment when the physical strength of the mouse was exhausted and it could not swim to the surface.
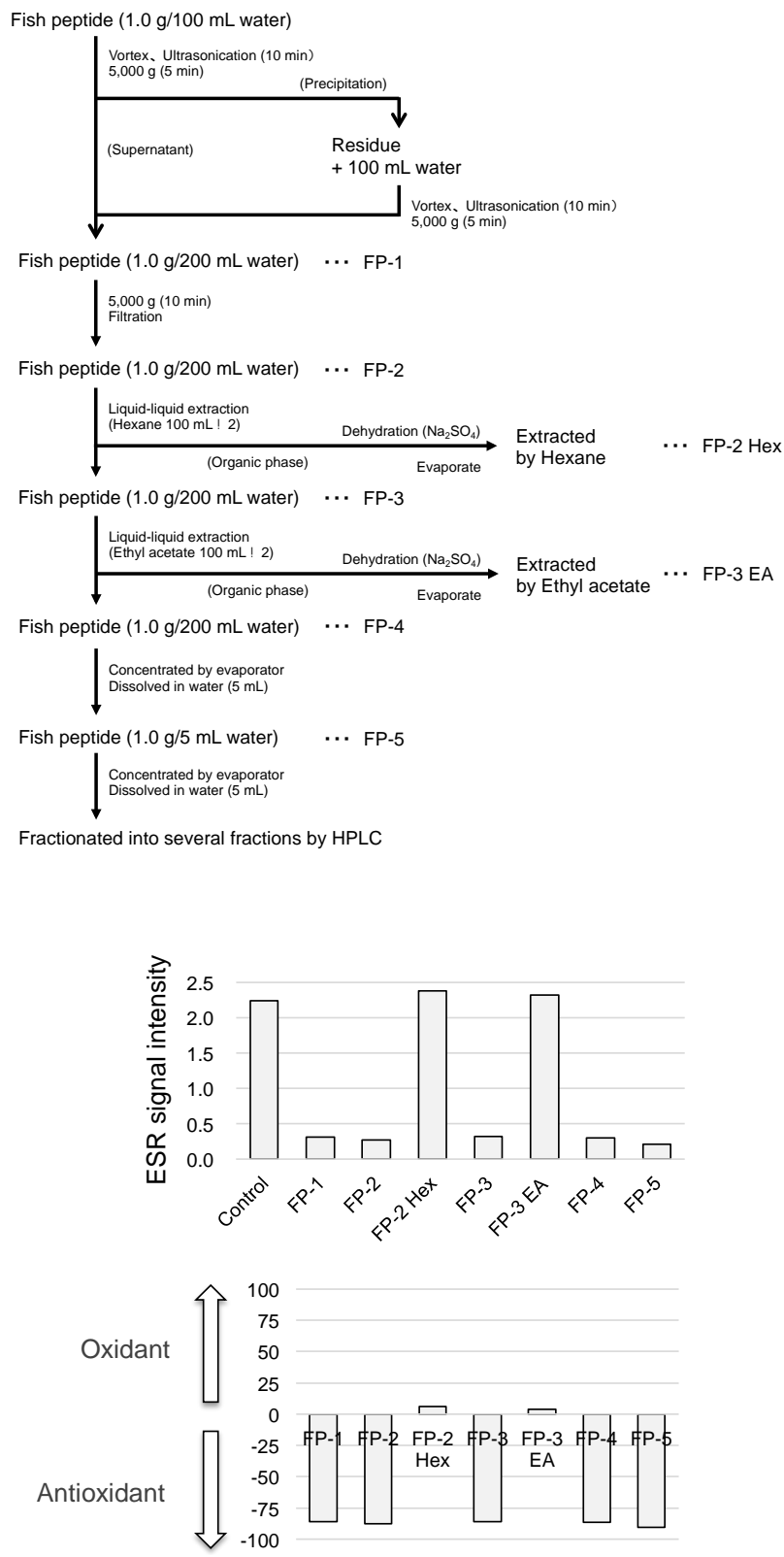

Figure 1. Diagram showing the sample preparation workflow and the procedures used for the measurement of antioxidant activities 


\subsection{Statistical Analysis}

Data are expressed as mean \pm SEM and statistical analyses were performed using GraphPad Prism 5 Statistical Software program (GraphPad Software for Science, San Diego, CA). Groups were analyzed via analysis of variance followed by Bonferroni and Dunn post hoc tests. A $P$ value less than 0.05 was considered statistically significant.

\section{Results}

\subsection{Fractionation of FMDP by HPLC}

Fish meat is contained in several types of amino acids and dipeptides [13]. The fish meat was purified with hexanes and ethyl acetate to remove lipids before the fish meat was fractionated using revised phase HPLC for the antioxidant activity assay. Each prepared sample was assessed for antioxidant activity via ESR. Although the organic phase fraction (FP-2 Hex and FP-3 EA) did not have antioxidant activity, the water phase (FP-5) had a strong antioxidant activity (Figure 1). Purified fish meat samples (FP-5) were analyzed using MS/MS and the spectra from the samples are shown in Figure 2. We obtained a mass spectrum of 278.4 as a protonated ion. We estimated RI or IR from the mass spectrum and fragmented ion. FP-5 was fractionated using HPLC. These HPLC fractional samples were assessed for antioxidant activity (Figure 3A), and antioxidant activity was detected in Fractions 1, 2, and 5. We obtained mass spectra for all of the fractions (Figure 3B). We assumed that Fractions 1 and 2 contained RI or IR because the protonated ion was 288.3 and fragment ions were $115.9\left(\mathrm{Ile}_{-} \mathrm{H}_{2} \mathrm{O}\right)$ and 174.9 (Arg), respectively.
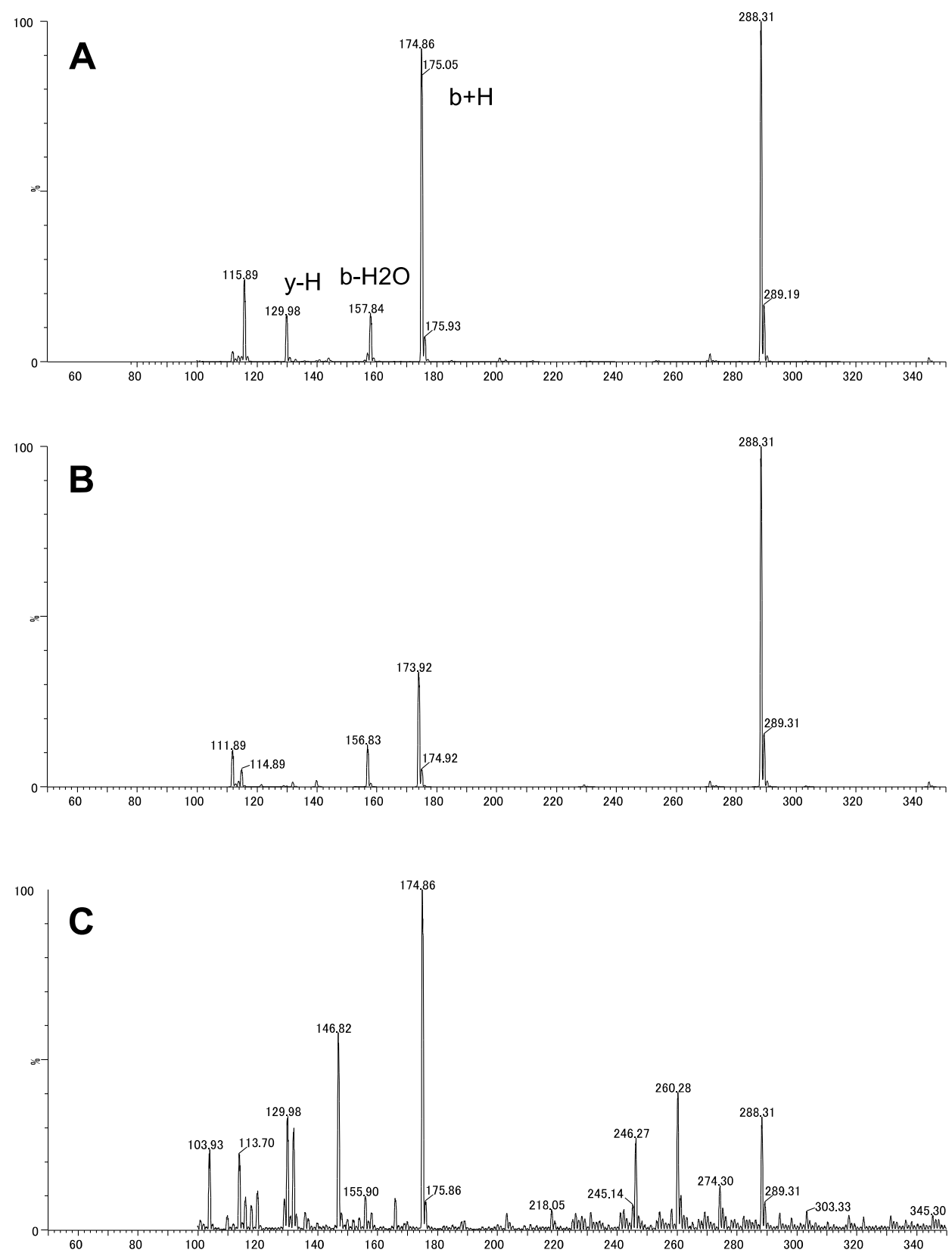
A

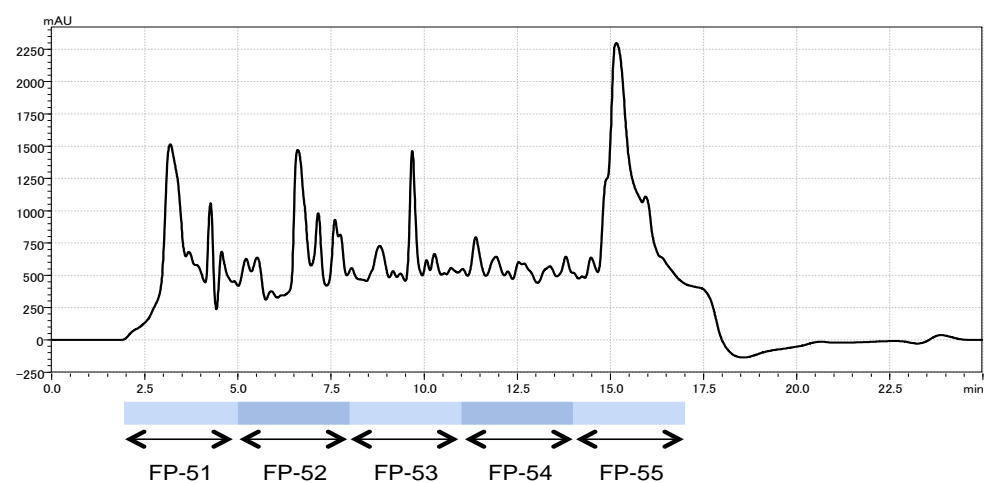

B

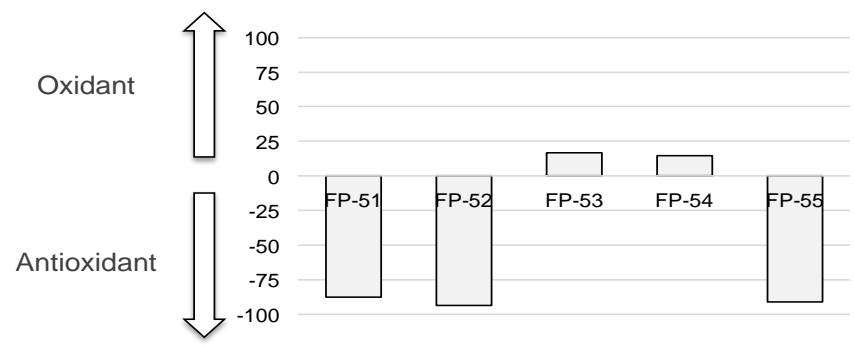

Figure 3. HPLC chromatogram of FP-5 and the antioxidant activities of the fractionated samples

Quantitative analysis of RI and IR was performed using hydrophilic interaction chromatography with tandem mass spectrometry (HILIC/MS/MS). We decided RI and IR peak using those standard solutions.

\subsection{Effects of FMDP, IR, and RI on the Body Weights of Mice}

The body weights were not changed after treatment of 100, $300 \mathrm{mg} / \mathrm{kg}$ FMDP, 1 and $3 \mathrm{mg} / \mathrm{kg}$ IR and RI compared with vehicle treatment (Figure 4A and Figure 4B). However, body weight was decreased after treatment with $15 \mathrm{mg} / \mathrm{kg}$ DEX. The DEX-induced loss of body weight was not changed after treatment with FMDP, IR, and RI (Figure 4C).
We performed a weighted forced swimming test to investigate the anti-fatigue effects of FMDP, IR, and RI. The ratio of the swim time on Day 2 divided by the swim time of Day 0 was used as an index of fatigue. The swim time of normal mice was not affected by the administration of FMDP, IR, or RI compared to the vehicle-treated group. (Figure 5A and Figure 5B). However, the administration of DEX once per day on Days 0-2 significantly reduced the swim time on Day 2. The DEX-induced decrease in swim time was significantly abolished following the administration of FMDP. Furthermore, IR or RI treatment produced a tendency toward increased swim time after the DEX-induced decrease in swim time (Figure 5C).
A
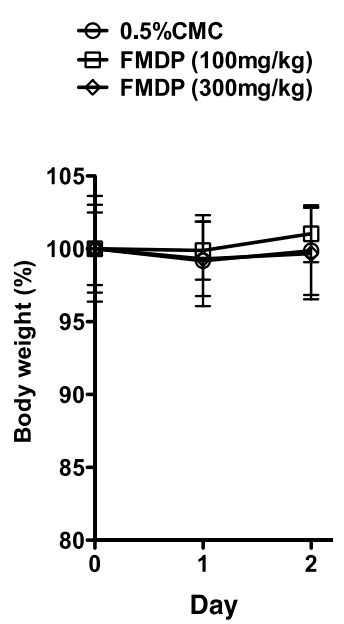

B

$$
\begin{aligned}
& \text { ๑ } 0.5 \% \mathrm{CMC} \\
& -1 \mathrm{mg} / \mathrm{kg} \mathrm{IR} \\
& -3 \mathrm{mg} / \mathrm{kg} \mathrm{IR} \\
& -1 \mathrm{mg} / \mathrm{kg} \mathrm{RI} \\
& -3 \mathrm{mg} / \mathrm{kg} \mathrm{RI}
\end{aligned}
$$

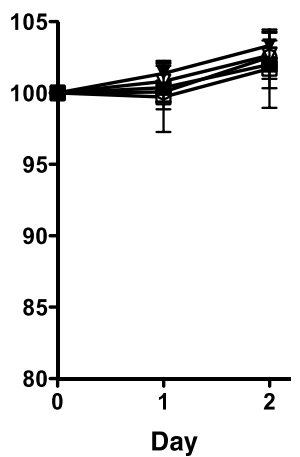

C
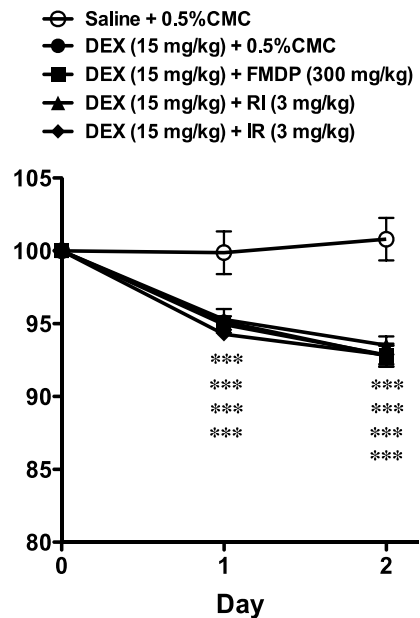

Figure 4. Effects of fish meat-derived peptide extract (FMDP), dipeptide (arginine-isoleucine; RI or isoleucine-arginine; IR) or the vehicle control (0.5\% carboxymethyl cellulose; $\mathrm{CMC}$ ) on the body weight of mice. Body weights were not significantly different among the $0.5 \% \mathrm{CMC}, 100 \mathrm{or} 300 \mathrm{mg} / \mathrm{kg}$ Ext., 1 or $3 \mathrm{mg} / \mathrm{kg}$ IR or RI groups (A and B). The effects of FMDP, RI and IR on the body weight of dexamethasone (DEX)-treated mice. The body weight was significantly decreased by DEX. The loss of body weight was not changed by the administration of $300 \mathrm{mg} / \mathrm{kg}$ FMDP, $3 \mathrm{mg} / \mathrm{kg}$ RI and IR (C). Each point represents the mean \pm S.E.M. of data from 8 to 10 mice. $* * * P<0.001$ vs. Saline $+0.5 \%$ CMC 
A

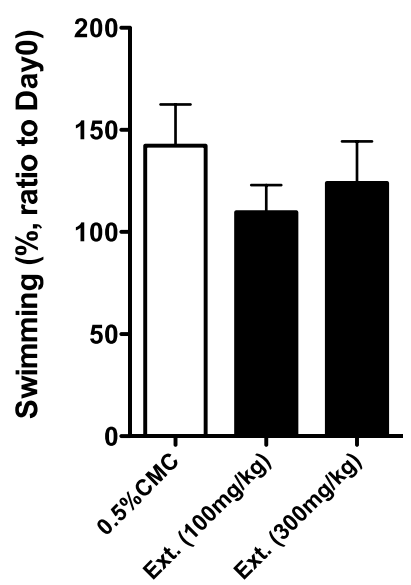

B

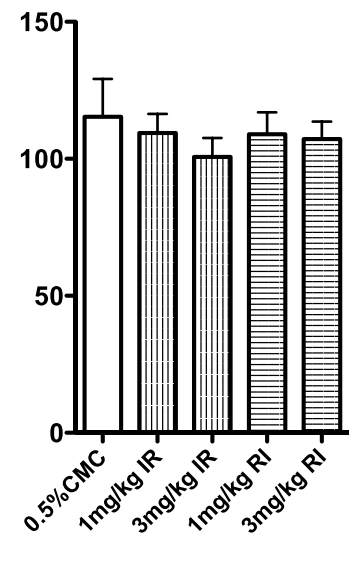

C

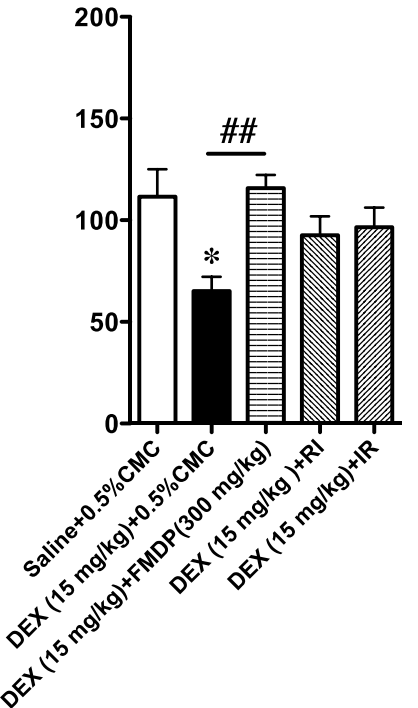

Figure 5. Effects of FMDP, IR, and RI on the weighted forced swimming in the naïve or DEX- treated mice. The swim time was not significantly different among the $0.5 \% \mathrm{CMC}, 100$ or $300 \mathrm{mg} / \mathrm{kg}$ FMDP, 1 or $3 \mathrm{mg} / \mathrm{kg}$ IR or RI treatment groups (A and B). The reduction of the swim time was significantly recovered after administration of $300 \mathrm{mg} / \mathrm{kg}$ FMDP (C). Each column represents the mean \pm S.E.M. of $8-10$ mice. $* P<0.05$ vs. Saline + $0.5 \%$ CMC. \#\# $P<0.01$ vs. DEX $(15 \mathrm{mg} / \mathrm{kg})+0.5 \% \mathrm{CMC}$

\section{Discussion}

Although many aspects of fatigue have been studied for a long time, no appropriate method has been established for the optimal and objective assessment of fatigue. The forced swim test is often used in mouse studies to measure fatigue [14-16]. In this study, we hypothesized that IR and RI are the peptides with the highest antioxidant activity in FMDP. However, the therapeutic mechanisms of IR and RI dipeptides on reducing fatigue are still unclear. In the present study, mice swam to fatigue, and the change in swim time after the administration of FMDP and dipeptides (IR and RI) was evaluated. This study suggests that reduction in swim time that is observed after DEX administration may be recovered by administration of FMDP, IR, and RI. However, these substances cannot increase the swim time of naive mice.

During DEX-induced muscular atrophy, there is an increase in muscle degradation and an inhibition of muscle synthesis, which leads to an atrophic state of the muscle tissue [17]. By suppressing Akt phosphorylation, mTORC1 is suppressed, and downstream proteins such as P70S6K and elf4E are suppressed, which reduces overall protein synthesis. $[18,19,20]$. The degradation process is triggered by increased transcription of REDD1 and REDD2 (regulated in development and DNA damage responses 1/2), which increase the activity of the myostatin/Smad2/3 pathway [17]. REDD1/2 are related to the stress response and are activated by glucocorticoid administration and increased ROS generation [21,22,23]. However, oxidative stress mainly promotes via the activation of p38 mitogen-activated protein kinase, which subsequently induces the expression of atrogin-1, MuRF1 and the autophagy-lysosome system [24,25]. Moreover, it also has been reported that DEX-induced muscle atrophy is partially caused by mitochondrial dysfunction mediated by the PGC- $1 \alpha /$ TFAM and PGC- $1 \alpha / \mathrm{mfn} 2$ signaling pathways [11]. The mechanism through which FMDP suppresses DEX-induced fatigue is not clarified in this study. Therefore, further research is necessary to identify this mechanism.

\section{Conclusions}

Our data suggest that FMDP, IR, and RI may help reduce on DEX-induced fatigue. However, comprehensive chemical and pharmacological research is required to determine the mechanism through which FMDP affects DEX-induced fatigue.

\section{Acknowledgements}

We thank Mr. Yuta Suzuki, Mr. Hiroto Takeuchi and Ms. Yui Harada for their technical assistance.

\section{Statement of Competing Interests}

The authors indicated no potential conflicts of interest.

\section{List of Abbreviations}

CMC: Carboxymethyl cellulose

DEX: Dexamethasone

ESR: Electron spin resonance

FMDP: Fish meat-derived peptide

HPLC: High-performance liquid chromatography

IR: Isoleucine-arginine

RI: Arginine-isoleucine

ROS: Reactive oxygen species

WHO: World Health Organization 


\section{References}

[1] World Health Organization. "World health statistics 2018 monitoring health for the SDGs", 2018.

[2] D'Autreaux, B. and Toledano, M.B. "ROS as signalling molecules: mechanisms that generate specificity in ROS homeostasis", Nat Rev Mol Cell Biol, 8, 813-824, 2007.

[3] Gao, X., Wilsgaard, T., Jansen, E., Xuan, Y., Anusruti, A., Brenner, H. and Schottker, B. "Serum total thiol levels and the risk of lung, colorectal, breast and prostate cancer: A prospective casecohort study", Int J Cancer, 2019.

[4] Mathis, K.W., Venegas-Pont, M., Masterson, C.W., Stewart, N.J., Wasson, K.L. and Ryan, M.J. "Oxidative stress promotes hypertension and albuminuria during the autoimmune disease systemic lupus erythematosus", Hypertension, 59, 673-679, 2012.

[5] Papada, E., Forbes, A., Amerikanou, C., Torovic, L. Kalogeropoulos, N., Tzavara, C., Triantafillidis, J.K. and Kaliora, A.C. "Antioxidative efficacy of a Pistacia lentiscus supplement and its effect on the plasma amino acid profile in inflammatory bowel disease: A randomised, double-blind, placebo-controlled trial", Nutrients, 10, 2018.

[6] Fukuda, S., Nojima, J., Motoki, Y., Yamaguti, K., Nakatomi, Y. Okawa, N., Fujiwara, K., Watanabe, Y. and Kuratsune, H. "A potential biomarker for fatigue: Oxidative stress and anti-oxidative activity", Biol Psychol, 118, 88-93, 2016

[7] Ramamoorthy, S. and Cidlowski, J.A. "Exploring the molecular mechanisms of glucocorticoid receptor action from sensitivity to resistance", Endocr Dev, 24, 41-56, 2013.

[8] Dardevet, D., Sornet, C., Taillandier, D., Savary, I., Attaix, D. and Grizard, J. "Sensitivity and protein turnover response to glucocorticoids are different in skeletal muscle from adult and old rats. Lack of regulation of the ubiquitin-proteasome proteolytic pathway in aging", J Clin Invest, 96, 2113-2119, 1995.

[9] Mitch, W.E. and Goldberg, A.L. "Mechanisms of muscle wasting. The role of the ubiquitin-proteasome pathway", N Engl J Med, 335, 1897-1905, 1996.

[10] Gupta, A. and Gupta, Y. "Glucocorticoid-induced myopathy: Pathophysiology, diagnosis, and treatment", Indian J Endocrinol Metab, 17, 913-916, 2013.

[11] Huang, Y., Chen, K., Ren, Q., Yi, L., Zhu, J., Zhang, Q. and Mi, M. "Dihydromyricetin attenuates dexamethasone-induced muscle atrophy by improving mitochondrial function via the PGC-1alpha pathway", Cell Physiol Biochem, 49, 758-779, 2018.

[12] Begum, G., Cunliffe, A. and Leveritt, M. "Physiological role of carnosine in contracting muscle", Int J Sport Nutr Exerc Metab, 15, 493-514, 2005

[13] Schindler, A., Dunkel, A., Stahler, F., Backes, M., Ley, J., Meyerhof, W. and Hofmann, T. "Discovery of salt taste enhancing arginyl dipeptides in protein digests and fermented fish sauces by means of a sensomics approach", J Agric Food Chem, 59, 12578 12588,2011

[14] Ohba, T., Domoto, S., Tanaka, M., Nakamura, S., Shimazawa, M. and Hara, H. "Myalgic encephalomyelitis/chronic fatigue syndrome induced by repeated forced swimming in mice", Biol Pharm Bull, 42, 1140-1145, 2019.

[15] Matsuoka, R., Kimura, M., Uno, S., Shidara, H. and Kunou, M. "Egg white hydrolysate improves fatigue due to short-term swimming load test in mice", Food Sci Nutr, 6, 2314-2320, 2018.

[16] Jiang, X., Chu, Q., Li, L., Qin, L., Hao, J., Kou, L., Lin, F. and Wang, D. "The anti-fatigue activities of Tuber melanosporum in a mouse model”, Exp Ther Med, 15, 3066-3073, 2018

[17] Schakman, O., Kalista, S., Barbe, C., Loumaye, A. and Thissen, J.P. "Glucocorticoid-induced skeletal muscle atrophy", Int J Biochem Cell Biol, 45, 2163-2172, 2013.

[18] Gordon, B.S., Kelleher, A.R. and Kimball, S.R. "Regulation of muscle protein synthesis and the effects of catabolic states", Int J Biochem Cell Biol, 45, 2147-2157, 2013.

[19] Bentzinger, C.F., Romanino, K., Cloetta, D., Lin, S., Mascarenhas, J.B., Oliveri, F., Xia, J., Casanova, E., Costa, C.F., Brink, M., Zorzato, F., Hall, M.N. and Ruegg, M.A. "Skeletal musclespecific ablation of raptor, but not of rictor, causes metabolic changes and results in muscle dystrophy", Cell Metab, 8, 411-424, 2008.

[20] Brugarolas, J., Lei, K., Hurley, R.L., Manning, B.D., Reiling, J.H., Hafen, E., Witters, L.A., Ellisen, L.W. and Kaelin, W.G., Jr. "Regulation of mTOR function in response to hypoxia by REDD1 and the TSC1/TSC2 tumor suppressor complex", Genes Dev, 18, 2893-2904, 2004.

[21] Katiyar, S., Liu, E., Knutzen, C.A., Lang, E.S., Lombardo, C.R., Sankar, S., Toth, J.I., Petroski, M.D., Ronai, Z. and Chiang, G.G. "REDD1, an inhibitor of mTOR signalling, is regulated by the CUL4A-DDB1 ubiquitin ligase", EMBO Rep, 10, 866-872, 2009.

[22] Ellisen, L.W., Ramsayer, K.D., Johannessen, C.M., Yang, A., Beppu, H., Minda, K., Oliner, J.D., McKeon, F. and Haber, D.A. "REDD1, a developmentally regulated transcriptional target of p63 and p53, links p63 to regulation of reactive oxygen species", Mol Cell, 10, 995-1005, 2002.

[23] Lin, L., Stringfield, T.M., Shi, X. and Chen, Y. "Arsenite induces a cell stress-response gene, RTP801, through reactive oxygen species and transcription factors Elk-1 and CCAAT/enhancerbinding protein", Biochem J, 392, 93-102, 2005.

[24] Braun, T.P. and Marks, D.L. "The regulation of muscle mass by endogenous glucocorticoids", Front Physiol, 6, 12, 2015.

[25] McClung, J.M., Judge, A.R., Powers, S.K. and Yan, Z. "p38 MAPK links oxidative stress to autophagy-related gene expression in cachectic muscle wasting", Am J Physiol Cell Physiol, 298, C542-549, 2010. 